\title{
Toscana Virus Infection In Catalonia (Spain)
}

\author{
Neus Cardeñosa, Diana Kaptoul, ${ }^{2}$ Pedro Fernández-Viladrich, ${ }^{2}$ Carles Aranda, ${ }^{3}$ \\ Fernando de Ory, ${ }^{4}$ Jordi Niubó, ${ }^{2}$ Pere Plans, ${ }^{1}$ Angela Domínguez, \\ Giovanni Fedele, ${ }^{4}$ Antonio Tenorio, ${ }^{4}$ and María Paz Sánchez-Seco ${ }^{4}$
}

\begin{abstract}
Toscana virus (TOSV), an arthropod-borne phlebovirus, is an important agent of acute meningitis and meningoencephalitis in the Mediterranean area. The epidemiology of the infection in humans in Catalonia is at present unknown. In this study, we found a seroprevalence of infection of $6 \%$, and 2 clinical cases were detected by serology and/or PCR.
\end{abstract}

Key Words: Toscana virus—Mediterranean—Catalonia—Meningitis—Meningoencephalitis.

\section{Introduction}

$\mathbf{T}$ OSCANA VIRUs (TOSV) (family Bunyaviridae, genus Phlebovirus) is an important agent of acute meningitis and meningoencephalitis in residents and visitors from Mediterranean countries (Charrel et al. 2005) A significant proportion of infection results in asymptomatic or pauci-symptomatic forms. TOSV is an arthropod-borne virus, transmitted by phlebotomine sand flies during the summer-fall period. Although the first case of TOSV infection reported from Spain occurred in a Swedish tourist after a visit to Catalonia (northeast of Spain) (Eitrem et al. 1991), since then no more cases have been published and no seroepidemiological studies have been carried out in this region.

Phlebotomine populations are represented in Catalonia mainly by Phlebotomus perniciosus, a known vector of TOSV (Verani et al. 1988), and Sergentomyia minuta, in which the genome of TOSV has been detected recently (Aranda and Eritja 1998, Charrel et al. 2006).

A study on TOSV seroprevalence in Granada (southern Spain) (Sanbonmatsu-Gámez et al. 2005), showed a $24.9 \%$ seroprevalence rate. With this knowledge, and taking into account that in both Catalonia and Granada a high number of phlebotomine sand flies can be found, and also that canine leishmaniasis remains an important veterinarian challenge (Aransay et al. 2004), a study of seroprevalence of the TOSV infection was carried out.
At this same time, hospitalized patients with suspected viral meningitis or meningoencephalitis were studied in one of Catalonia's main hospitals, the Bellvitge Universitary Hospital, which is a teaching hospital for adult patients only and located in the Costa Ponent area.

\section{The Study}

Sera were obtained from a representative sample from Catalonia in 2002 consisting of healthy individuals aged 15 years or older. To calculate the sample size, the following assumptions were made: An estimated seroprevalence of $10 \%$ based in previous studies (Sanbonmatsu-Gámez et al. 2005, de Ory-Manchón et al. 2007), 95\% confidence level, and 2.5\% precision level. The minimum sample size was 833 . The population studied was stratified by sex, age, and geographic area and was representative of each area. Eight different areas were established taking into account location and altitude (Fig. 1A). Anti-TOSV immunoglobulin G (IgG) was detected by a commercial enzymatic immunoassay, the EIA Enzywell Toscana virus IgG (Diesse, Italy), adhering to the manufacturer's instructions.

Anti-TOSV IgG was analyzed in 833 human serum samples of the general population. The study population was distributed by geographic area proportional to that of Catalonia. By sex, $370(44.4 \%)$ were males and $463(55.6 \%)$ were females. By age groups, $174(20.9 \%)$ were $<31$ years, $513(61.6 \%)$ were

\footnotetext{
${ }^{1}$ Subdirecció General de Vigilància I Resposta a Emergències de Salut Pública, Direcció General de Salut Pública, Departament de Salut de Catalunya, Barcelona, Spain; General Directorate of Public Health of Catalonia, Barcelona, Spain.

${ }^{2}$ IDIBELL Hospital Universitari de Bellvitge, Barcelona, Spain.

${ }^{3}$ Consell Comarcal del Baix Llobregat, Servei de Control de Mosquits, Barcelona, Spain.

${ }^{4}$ Laboratorio de Arbovirus y Enfermedades Víricas Importadas, Servicio de Microbiología Diagnóstica, Centro Nacional de Microbiología, Instituto de Salud Carlos III, Madrid, Spain.
} 
A

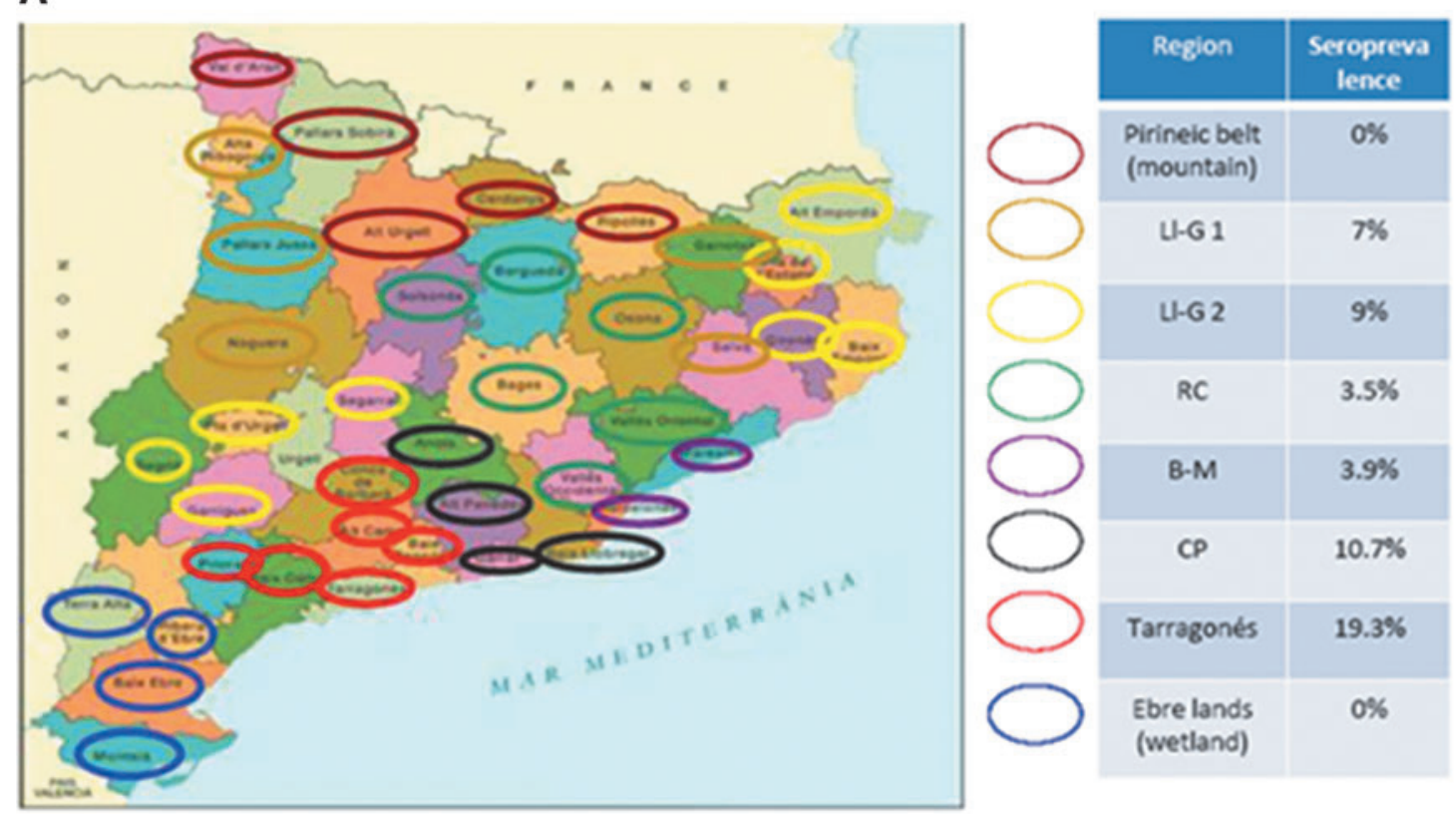

B

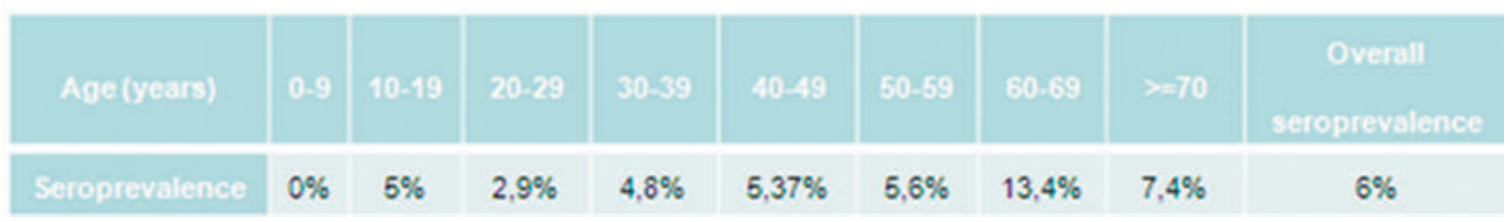

FIG. 1. The eight areas established for the seroprevalence study, taking into account location and altitude. (A) The seroprevalence in each region. (B) The level of seroprevalence in each group of age.

aged 31-60 years, and $146(17.5 \%)$ were $>60$ years. Fifty samples showed IgG-positive ratios, with an overall seroprevalence of $6 \%$. Anti-TOSV IgG was more often detected in persons $>60$ years (odds ratio [OR] 2.79, 95\% confidence interval $[\mathrm{CI}] 1.46-5.30, p=0.001)$ with a seroprevalence of $12.3 \%$ in this age group. Persons that showed signs of being IgG positive were equally divided by sex. When the different geographical areas were compared, seroprevalence rates ranged from $0 \%$ in the regions of the Pyrenean belt and Ebre lands (higher and lower altitudes), followed by $3.5 \%$ and $3.9 \%$, respectively, in the regions of Central Catalonia (RC) and Barcelona metropolitan area (B-M), 7\% in Lleida-Gironès region above 1000 meters (Ll-G 1), 9\% in Lleida-Gironès region below 1000 meters (Ll-G 2), 10.7\% in Costa Ponent (CP), and $19.3 \%$ in the region of Tarragona (Fig. 1A).

Fifty-five adult patients with suspected viral meningitis or meningoencephalitis, hospitalized from May, 2003, to November, 2005, in the Bellvitge hospital were studied. A sample of cerebrospinal fluid (CSF) was obtained from all patients; acute serum was obtained in 54 patients, and convalescent sera were obtained in the same number of people. Sera were studied with the described method. CSF samples were analyzed by a RT-nested-PCR for arboviruses, which combines three generic RT-nested-PCRs for the detection of alpha-, flavi-, and/or pheboviruses (Sánchez-Seco et al. 2010). AntiTOSV IgG-positive ratios, indicating past infections, were obtained in $12.7 \%$ of the patients. In 2 patients $(3.7 \%)$, IgM antibodies were detected, accompanied by IgG in both and a positive phlebovirus RT-nested PCR in one. The IgM-reactive patients were regarded as TOSV infections. One sample (1/ $55 ; 1.8 \%$ ) was reactive employing the generic phlebovirus RT-nested-PCR.

A 25-year-old man was admitted to the hospital in July, 2004, with a stiff neck, vomiting, and a fever (body temperature of $37.4^{\circ} \mathrm{C}$ ). He reported fever and headaches during the previous $48 \mathrm{~h}$. Hematologic and biochemical blood tests showed no abnormalities. A lumbar puncture analysis revealed a clear CSF with 88 white blood cells (WBCs) $/ \mathrm{mL}(89 \%$ lymphocytes), $100 \mathrm{mg} / \mathrm{dL}$ of total protein, and $57 \mathrm{mg} / \mathrm{dL}$ glucose. The patient's course of infection was benign and selflimited, and he received only supportive treatment during $48 \mathrm{~h}$. He was discharged 3 days after admission. TOSV PCR amplification on the CSF was negative. Acute and convalescent samples were positive for anti-TOSV-specific IgM and IgG.

The second patient was a 61-year-old woman who was admitted to the hospital in August, 2005. She suffered from diabetes mellitus type II and reported to be following the prescribed treatment. She reported fever $\left(38.4^{\circ} \mathrm{C}\right)$, headaches, a slight stiff neck, and repetitive vomiting that started $24 \mathrm{~h}$ prior to admission. Skin and abdomen were normal, as were cardiopulmonary and neurologic functions. WBC count was 12,400 cells $/ \mathrm{mL}$ ( $87 \%$ neutrophils). Blood fibrinogen was $5.8 \mathrm{~g} / \mathrm{L}$, and blood glucose was $234 \mathrm{mg} / \mathrm{dL}$. Other 
hematological and biochemical blood tests were normal. A lumbar puncture analysis revealed clear CSF, with $28 \mathrm{WBCs}$ / $\mathrm{mL}$ (differential WBC count was not done), $72 \mathrm{mg} / \mathrm{dL}$ proteins, and $122 \mathrm{mg} / \mathrm{dL}$ glucose. CSF Gram stain and culture were negative. The patient course of infection was benign and self-limited, and she received only supportive treatment for $48 \mathrm{~h}$. She was discharged 3 days after admission. No neurologic sequelae were observed 1 month after discharge. TOSV PCR amplification on the CSF was positive. Sequencing of the amplified fragment showed that the sequence was similar to the ones of Spanish (B) genotype (data not shown; accession number JQ753317). Acute samples were negative for anti TOSV-specific IgM and IgG. Convalescent samples were positive demonstrating seroconversion.

\section{Conclusions}

TOSV and possibly TOSV-related phleboviruses circulate in Catalonia, with an overall seroprevalence value similar to that reported previously in Madrid, central Spain, (de OryManchón et al. 2007) and lower than that found in Granada, south of Spain (Sanbonmatsu-Gámez et al. 2005). These values have been obtained with the same methodology, so they can be compared. The high seroprevalence rate found in Granada (25\%), similar to that reported in endemic areas of Italy (Charrel et al. 2005), is similar to the $19.3 \%$ found in the area of Tarragones, indicating a higher circulation of the virus and suggesting that cases of TOSV or TOSV-related phlebovirus infection could be misdiagnosed in this area. In fact, the seroprevalence rate obtained could be due to the cocirculation of TOSV and the recently described Granada virus (GRV) because they cross-react (Collao et al. 2010) and sequences similar to the ones of GRV were detected in the Costa Ponent area (Baix Llobregat) (Sánchez-Seco et al. 2010). However, because GRV and Massilia virus (MASV) (Charrel et al. 2009), other members of the Naples complex, have been described recently and more specific tests such as plaque neutralization are very complex within this genus, all seroprevalence data reported until now should not be considered specific for TOSV.

Analysis of the sequence of TOSV found in the described clinical case shows that the Spanish genotype (B) is circulating in Catalonia. This region is close to Marseille region where cocirculation of Spanish and Italian genotypes has been described (Charrel et al. 2007). More data about TOSV strains should be obtained to know if the Pyrenees act as a natural border between both genotypes.

Despite increasing evidence of the role of TOSV as a major cause of aseptic meningitis, meningoencephalitis, and encephalitis during the warm seasons in countries in which it circulates, the epidemiology of TOSV in Catalonia is still unknown, and few physicians are aware of its potential to cause central nervous system infections. TOSV infections in Catalonia, including neurological cases, may have remained undetected.

\section{Author Disclosure Statement}

No competing financial interests exist.

\section{References}

Aranda C, Eritja R. Phlebotominae (Diptera:Psychodidae) of the Baix Llobregat, Barcelona, Spain. Acta Parasitológica Portuguesa 1998; 5:28.

Aransay A, Testa J, Morillas-Márquez F, Lucientes J, et al. Distribution of sandfly species in relation to canine leishmaniasis from the Ebro Valley to Valencia, northeastern Spain. Parasitol Res 2004; 94:416-420.

Charrel RN, Gallian P, Navarro-Mari JM, Nicoletti L, et al. Remergence of Toscana virus in Europe. Emerg Infect Dis 2005; 11:1657-1663.

Charrel RN, Izri A, Temmam S, de Lamballerie X, et al. Toscana virus RNA in Sergentomyia minuta files. Emerg Infect Dis 2006; 12:1299-1300.

Charrel RN, Izri A, Temmam S, Delaunoy P, et al. Cocirculation of 2 genotypes of Toscana virus, southeastern France. Emerg Infect Dis 2007; 13:465-468.

Charrel RN, Moureau G, Temmam S, Izri A, et al. Massilia virus, a novel Phlebovirus (Bunyaviridae) isolated from sandflies in the Mediterranean. Vector Borne Zoonotic Dis 2009; 9: 519-530.

Collao X, Palacios G, de Ory F, Sanbonmatsu S, et al. Granada virus: A natural phlebovirus reassortant of the sandfly fever Naples serocomplex with low seroprevalence in humans.Am J Trop Med Hyg 2010; 83:760-765.

de Ory-Manchón F, Sanz-Moreno JC, Aránguez-Ruíz E, Fernández-Ramírez R. Age-dependent seroprevalence of Toscana virus in the Community of Madrid: 1993-1994 and 1999-2000. Enferm Infecc Microbiol Clin 2007; 25:187-189.

Eitrem R, Niklasson B, Weiland O. Sandfly fever among Swedish tourists. Scand J Infect Dis 1991; 23:451-457.

Sanbonmatsu-Gámez S, Pérez-Ruiz M, Collao X, Sánchez-Seco MP, et al. Toscana virus in Spain. Emerg Infect Dis 2005; 11:1701-1707.

Sánchez-Seco MP, Vázquez A, Collao X, Hernández L, et al. Surveillance of Arboviruses in Spanish Wetlands. Detection of New Flavi- and Phleboviruses. Vector Borne Zoonotic Dis. 2010; 10:203-206.

Verani P, Ciufolini MG, Caciolli S, Renzi A, et al. Ecology of viruses isolated from sand flies in Italy and characterized of a new Phlebovirus (Arabia virus). Am J Trop Med Hyg 1988; 38:433-439.
Address correspondence to: María Paz Sánchez-Seco National institute of Health "Carlos III" Ctral Pozuelo-Majadahonda km 2 28220 Madrid Spain

E-mail: paz.sanchez@isciii.es 